\title{
On the pro-representability of a functor on the category of finite group schemes
}

\author{
By
}

Masayoshi MiYanishI

(Received September 26, 1966)

\section{Part I. Preliminaries}

1. Let $S$ be a locally noetherian prescheme and let $G, X$ be respectively a group prescheme over $S$, a prescheme over $\mathrm{S}$ on which $G$ operates from the left, that is to say, there is a $S$ morphism $\sigma: G \underset{S}{\times} X \rightarrow X$ such that the following diagrams are commutative.
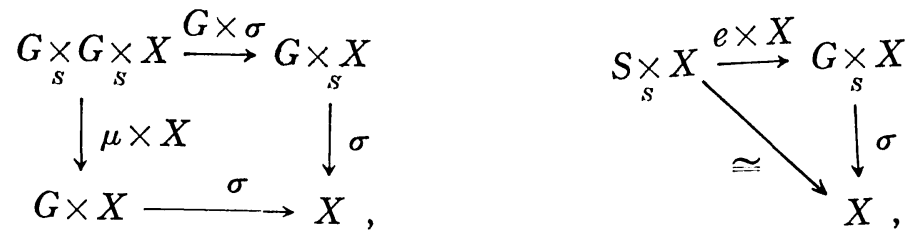

where $\mu$ (resp. $e$ ) is the multiplication (resp. the unit) of $G$. Then a diagram

$(*)$

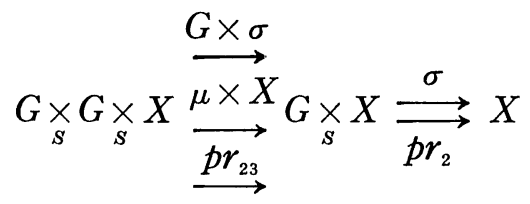

is a (Sch/S)-groupoid. In particular, if $G$ is a finite, locally free group scheme over $S$, we have the next result for the admissibility of the pre-equivalence relation $(*)$ (i.e. the existence of a geometric quotient of $X$ by $G$.)

Proposition 1. (Grothendieck-Gabriel, [1].) Let G be a finite, 
locally free group scheme over $S$. Suppose the next condition is satisfied in $(*)$ :

For any point $x \in X$, the set $\sigma(G \times x)$ (i.e. the orbit of $x)$ is contained in an affine open set of $X$.

Then we have:

(i ) There exists a cokernel $(Y, p)$ of $\left(\sigma, p r_{2}\right)$ in $(S c h / S)$, moreover such $a(Y, p)$ is a cokernel of $\left(\sigma, p r_{2}\right)$ in the category of all the ringed spaces.

(ii) The morphism $\left(\sigma, p r_{2}\right)_{Y}: G \underset{S}{\times} X \rightarrow \underset{Y}{\times} X$ is surjective. From (i) and (ii), $(Y, p)$ is a geometric quotient of $X$ by $G$, (the terminology is due to D. Mumford, [5].)

(iii) $p$ is integral, and $Y$ is affine if $X$ is affine.

(iv) If $\left(\sigma, p r_{2}\right)$ is a closed immersion (then, we call the operation $\sigma$ faithful), then $\left(\sigma, p r_{2}\right)_{Y}: G \underset{S}{\times} X \rightarrow X \underset{Y}{\times} X$ is an isomorphism, and $p$ is finite, locally free. If the rank of $G$ over $S$ is constant, the rank of $X$ over $Y$ is equal to the rank of $G$ over $S$.

Remark. If there exists an affine morphism $p^{\prime}: X \rightarrow Y^{\prime}$ such that $p^{\prime} \cdot \sigma=p^{\prime} \cdot p r_{2}$, then the above condition is satisfied.

Corollary. In the situation of Proposition 1, if $G$ operates faithfully on $X,(Y, p)$ is a universal geometric quotient, i.e. for any morphism $Y^{\prime} \rightarrow Y,\left(Y^{\prime}, p_{Y^{\prime}}\right)$ is a geometric quotient of $X \underset{Y}{\times} Y^{\prime}$ by $G$.

Proof. From (i), (iv) of Proposition 1, we know that $X \underset{Y}{X} \underset{\rightarrow}{\rightarrow}$ $G \underset{S}{\times} X$ i.e. if we change the base from $Y$ to $X, X$ becomes trivial and that $p: X \rightarrow Y$ is a faithfully flat, quasi-compact morphism. Note that for any morphism $f: Y^{\prime} \rightarrow Y$, the morphism $p_{Y^{\prime}}$ : $X \underset{Y}{X} Y^{\prime} \rightarrow Y^{\prime}$ is also a faithfully flat, quasi-compact morphism. We put $X^{\prime}=X \underset{Y}{\times} Y^{\prime}, p^{\prime}=p_{Y^{\prime}}$ and $\sigma^{\prime}=\sigma_{Y^{\prime}}$. Since $p^{\prime}$ is affine, from the remark, we know the existence of a geometric quotient $\left(Y^{\prime \prime}, q^{\prime}\right)$ of $X^{\prime}$ by $G$,

$$
G \underset{s}{\times} X^{\prime} \underset{p r_{2}}{\stackrel{\sigma^{\prime}}{\longrightarrow}} X^{\prime} \stackrel{q^{\prime}}{\longrightarrow} Y^{\prime \prime} \stackrel{q}{\longrightarrow} Y^{\prime}, \quad q \cdot q^{\prime}=p^{\prime} .
$$


Now, we extend the base $Y^{\prime}$ to $X^{\prime}$ by a morphism $p^{\prime}: X^{\prime} \rightarrow Y^{\prime}$, then we have the following commutative diagram,

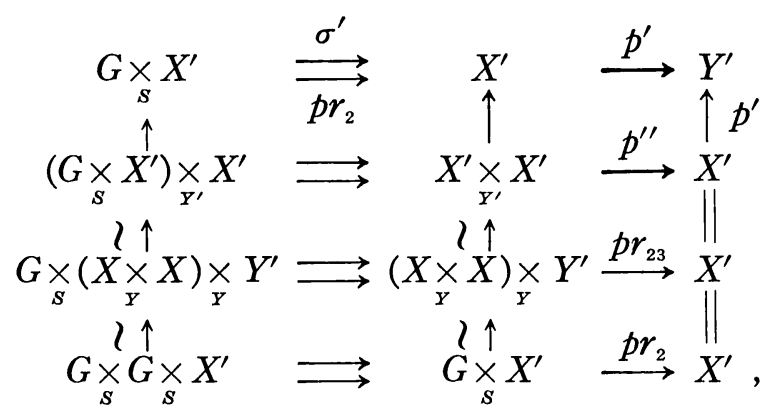

where in the lower row, $G$ operates on $G \underset{S}{\times} X^{\prime}$ through the multiplication of $G$, therefore $X^{\prime}$ is a geometric quotient of $G \times X_{S}^{\prime}$ by $G$. Therefore, $\left(X^{\prime}, p^{\prime \prime}\right)$ is a geometric quotient in the second row. On the other hand, we have

$$
p^{\prime \prime}=p_{X^{\prime}}^{\prime}=q_{X^{\prime}} \cdot q_{X^{\prime}}^{\prime}: X^{\prime} \underset{Y^{\prime}}{\times} X^{\prime} \stackrel{q_{X^{\prime}}^{\prime}}{\longrightarrow} Y^{\prime \prime} \underset{Y^{\prime}}{\times} X^{\prime} \stackrel{q_{X^{\prime}}}{\longrightarrow} X^{\prime} .
$$

Hence $q_{X^{\prime}}: Y_{Y^{\prime}}^{\prime \prime} X^{\prime} \rightarrow X^{\prime}$ is isomorphism. Since $p^{\prime}: X^{\prime} \rightarrow Y^{\prime}$ is faithfully flat, quasi-compact, we have, $Y^{\prime \prime} \underset{q}{\sim} Y^{\prime}$. (cf. [2], Exp. VIII, Cor. 5. 4.)

q.e.d.

2. We denote by $C_{f}(S)$ the category of finite, locally free group schemes over $S$. Let $G \in C_{f}(S)$ and let $X, Y$ be pre-schemes over $S$ such that $G$ operates faithfully on $X$ and that $Y$ is a geometric quotient of $X$ by $G$. In this situation, we will say formally that a sequence $G \underset{S}{\times} X \rightarrow X \rightarrow Y$ is exact.

Let us fix a group scheme $G \in C_{f}(S)$ and a pre-scheme $Y$ over $S$, and denote by $E_{S}^{\prime}(G, Y)$ the set of all pairs $(X, p)$ of a prescheme $X$ over $S$ and a $S$-morphism $p: X \rightarrow Y$ such that $G \underset{S}{\times} X \rightrightarrows$ $X \underset{p}{\rightarrow} Y$ is exact.

Lemma 1. Let $Y$ be a pre-scheme of finite type over $S$, and let $(X, p),\left(X^{\prime}, p^{\prime}\right)$ be two elements of $E_{S}^{\prime}(G, Y)$. Suppose there exists a morphism $f: X \rightarrow X^{\prime}$ such that $p^{\prime} \cdot f=p$ and that $f$ commutes with 
the operations of $G$ on $X$ and $X^{\prime}$, i.e. $\sigma^{\prime} \cdot(G \times f)=f \cdot \sigma$. Then $f$ is an isomorphism.

Proof. It is immediate to see that the underlying topological spaces of $X$ and $X^{\prime}$ is bijective under $f$, (cf. Lemma 1 of Part II). We have only to show the isomorphism of $O_{X}$ and $\mathcal{O}_{X^{\prime}}$. By virtue of Corollary to Proposition 1, we can suppose that $Y=\operatorname{Spec}(B)$, $X=\operatorname{Spec}(A), X^{\prime}=\operatorname{Spec}\left(A^{\prime}\right), f=\operatorname{Spec}(\varphi)$, where $B$ is a noetherian local ring, $A, A^{\prime}$ are $B$-algebras which are free $B$-modules with the same finite rank, and $\varphi$ is a homomorphism of $B$-algebras. The image $\varphi\left(A^{\prime}\right)$ of $\phi$ defines a closed image $X^{\prime \prime}$ of $f$ in $X^{\prime}$, and it is easy to see that $X^{\prime \prime}$ belongs also to $E_{S}^{\prime}(G, Y)$ and that $X \rightarrow$ $X^{\prime \prime}, X^{\prime \prime} \rightarrow X^{\prime}$ are morphisms of $G$-pre-schemes. Therefore, we can assume that $\varphi$ is surjective or injective. Next we change the base $Y$ to $\operatorname{Spec}(B / \mathfrak{m}), \mathfrak{m}$ : the maximal ideal of $B$. Then $A^{\prime} / \mathfrak{m} A^{\prime}, A / \mathfrak{m} A$ are vector spaces of the same dimension over $B / \mathrm{m}$. The same argument as for $Y=\operatorname{Spec}(B)$ shows that $A^{\prime} / \mathrm{m} A^{\prime} \sim A / \mathrm{m} A$. If $\phi$ is injective, we have $A=A^{\prime}+\mathfrak{m} A$, and by Nakayama's lemma, we have $A^{\prime} \sim \mathcal{A} A$. If $\varphi$ is surjective, since $A$ is $B$-projective module, $A^{\prime} \cong A \oplus M$, for some $B$-module $M$. Since $A^{\prime} / \mathfrak{m} A^{\prime} \cong A / \mathfrak{m} A$, we have $\mathfrak{m} M=M$. Hence $M=0$. q.e.d.

Corollary. Let $G, Y$ be as in Lemma 1 , and let $X$ be an element of $E_{S}^{\prime}(G, Y), G \underset{S}{X \underset{p}{\rightarrow}} \underset{\vec{p}}{\vec{\sigma}} X \underset{p}{\rightarrow} Y$. If phas a section s (i.e. S-morphism $p r_{2}$

$Y \rightarrow X$ such that $p \cdot s=Y)$, then $X$ is isomorphic to $G \times \underset{S}{\times} Y$.

Thus two elements $(X, p),\left(X^{\prime}, p^{\prime}\right)$ of $E_{S}^{\prime}(G, Y)$ are isomorphic if there exists a morphism $f: X \rightarrow X^{\prime}$ such that $p^{\prime} \cdot f=p$ and that $f$ commutes with the operations of $G$ on $X$ and $X^{\prime}$. Then, this defines an equivalence relation in $E_{S}^{\prime}(G, Y)$ by virtue of Lemma 1. We denote by $E_{S}(G, Y)$ the quotient of $E_{S}^{\prime}(G, Y)$ by the above equivalence relation.

Proposition 2. Let $f: Z \rightarrow Y$ be a S-morphism. We associate to any element $X \in E_{S}^{\prime}(G, Y)$ an element $X \underset{Y}{ } Z$ of $E_{S}^{\prime}(G, Z)$, (well defined). Then this mapping defines a mapping $f^{*}: E_{S}(G, Y) \rightarrow$ $E_{S}(G, Z)$. 
Proof. We omit the proof.

q.e.d.

Proposition 3. Let $Y$ be a scheme over $S$ and let $\alpha: G \rightarrow H$ be a homomorphism of finite, locally free group schemes over $S$, which satisfy the condition:

$(S)$ : the kernel of $\alpha: G \rightarrow H$, i.e. $K=G \times \underset{H}{X}$ is locally free.

We define an operation $\sigma^{\prime}$ of $G$ on $H \underset{S}{\times} X$ by $\sigma^{\prime}=\left(\mu_{H} \cdot \tau \cdot(\iota \alpha \times H) \cdot p r_{12}\right.$, $\left.\sigma \cdot p r_{13}\right)$, where $\mu_{H}, \tau, \sigma, \iota$ are respectively the multiplication of $H$, the exchange of two members, the operation of $G$ on $X$ and the inverse morphism of $G$. Then there exists a geometric quotient $X^{\prime}$ of $H \times \underset{S}{\times}$ by $G$ on which $H$ operates faithfully and which has $Y$ as a geometric quotient by $H$. In particular, if $H=G / K, X^{\prime}$ is obtained as a geometric quotient of $X$ by $K$ (restricting the operation of $G$ to $K$ ). If $\alpha: G \rightarrow H$ is a closed immersion, then the canonical morphism $X \rightarrow X^{\prime}$ is also a closed immersion. In this situation, $\alpha$ defines a mapping $\alpha_{*}: E_{S}(G, Y) \rightarrow E_{S}(H, Y)$.

Proof. The proof consists of several steps.

(I) The existence of $X$. We will begin by showing that the $G$ on $H \underset{S}{ } \times X$ is faithful. The operation is decomposed as follows:

$$
\begin{aligned}
& G \times H \times X \underset{\tau \times X}{\underset{\longrightarrow}{\sim}} H \times G \times X \underset{H \times \Delta_{G / S} \times X}{\longrightarrow} H \times G \times G \times X \underset{H \times \alpha \times G \times X}{\vec{X}} \\
& H \times H \times G \times X \underset{H \times \iota \times G \times X}{\longrightarrow} H \times H \times G \times X \frac{}{\left(\mu_{H}, H\right) \times(\sigma, X)} \\
& H \times H \times X \times X \underset{H \times \tau \times X}{\stackrel{T}{H}} H \times X \times H \times X,
\end{aligned}
$$

and all morphisms except $(H \times \alpha \times G \times X) \cdot\left(H \times \Delta_{G / S} \times X\right)$ are closed immersions. Therefore, we have only to prove that $(\alpha \times G) \cdot \Delta_{G / S}$ is a closed immersion. Then by EGA, (I. 4. 2.3.) and (I. 4.2.4), the unit morphism $S \rightarrow H$ is a closed immersion, hence $K \rightarrow G$ is a closed immersion. In this case, using the condition ( $S$ ), there exists a geometric quotient $G / K$ and $\alpha$ is decomposed to $\beta \cdot \alpha^{\prime}: G \underset{\alpha^{\prime}}{\rightarrow} G / K \rightarrow \vec{\beta} H$. Here, $\beta$ is also a closed immersion. Then we have $(\alpha \times G) \Delta_{G / S}=(\beta \times G)\left(\alpha^{\prime} \times G\right) \Delta_{G / S}$. Since $(\beta \times G)$ is a closed immersion, we can assume that $H=G / K$. Then the base change 
$G \stackrel{\alpha}{\rightarrow} H$, applied to a $H$-morphism $G \underset{\Delta_{G / S}}{\longrightarrow} G \times G \underset{\alpha \times G}{\longrightarrow} H \times G$ gives us a closed immersion, $K \times G \stackrel{\widetilde{\rightarrow}}{\rightarrow} \underset{H}{ } \times \rightarrow \rightarrow K \times G \times G \rightarrow G \times G$ (formally, $(k, g) \rightarrow(k, g, g) \rightarrow(k g, g))$, which is equal to $K \times G \underset{i \times G}{\longrightarrow} G \times G \underset{\left(\mu_{G}, p r_{2}\right)}{\sim}$ $G \times G$. Since $G \underset{\alpha}{\rightarrow} H$ is faithfully flat, quasi-compact, $(\alpha \times G) \Delta_{G / S}$ is a closed immersion, (cf. [2], Exp. VIII, Cor. 5.5). Next, $H \underset{S}{\times} \underset{(\pi \times p)}{\longrightarrow} \underset{S}{\longrightarrow} Y \stackrel{\sim}{\rightarrow} Y$ is affine and invariant with respect to the operation $\sigma^{\prime}$ of $G$ on $H \times X$. Therefore there exists a geometric quotient $X^{\prime}$ of $H \underset{S}{\times} X$ by $G$.

(II) $H$ operates faithfully on $X$. The operation $\sigma^{\prime \prime}$ of $H$ on $X^{\prime}$ is defined from the next commutative diagram,

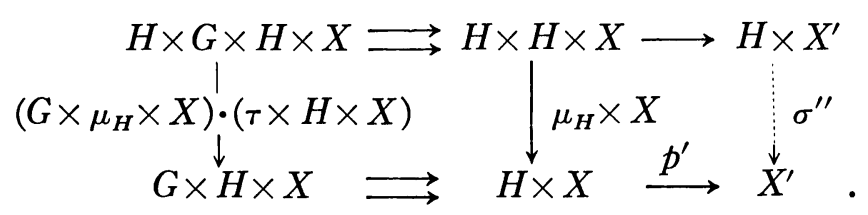

Consider the following commutative diagram, all objects considered naturally defined over $Y$,

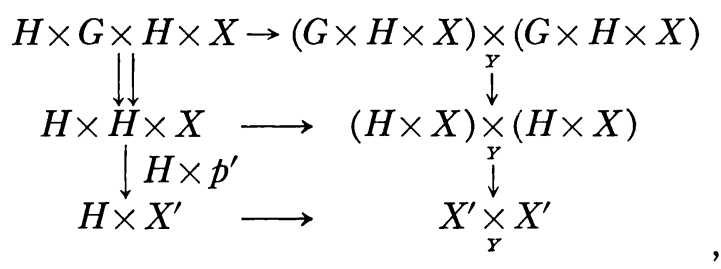

where it is easy to point out the morphisms. The base change by a faithfully flat, quasi-compact morphism $p: X \rightarrow Y$ gives us the following commutative diagram,

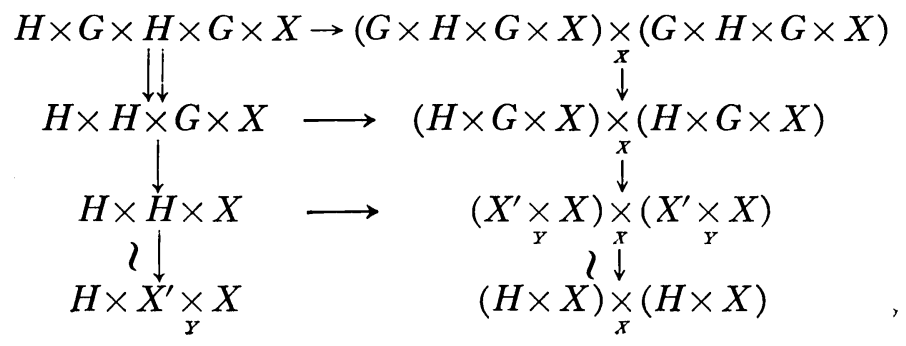


where we have used the fact that $X$ is an universal geometric quotient to prove that $X^{\prime} \times \underset{Y}{\sim} \underset{\rightarrow}{\rightarrow} \times X$. It is easy to point out the morphisms of the above diagram. Therefore $\left(H \times X^{\prime}\right) \times X$ is isomorphic to $\left(X^{\prime} \underset{Y}{\times} X^{\prime}\right) \underset{Y}{\times} X$. Hence an isomorphism $\underset{S}{\times} X^{\prime} \stackrel{\sim}{\rightarrow} X_{Y}^{\prime} \underset{Y}{\times} X^{\prime}$. Since $Y$ is a scheme over $S$, a morphism $X^{\prime} \times \underset{Y}{ } \underset{X^{\prime}}{\rightarrow}\left(X^{\prime} \times X^{\prime}\right) \underset{Y \times Y}{\times}$ $\left(Y, \Delta_{Y / S}\right) \rightarrow X^{\prime} \times X^{\prime} \cong\left(X_{S}^{\prime} \times X^{\prime}\right)_{Y \times Y}(Y \times Y)$ is a closed immersion. Therefore $H \times X_{S}^{\prime} \rightarrow X^{\prime} \times X_{S}^{\prime}$ is a closed immersion.

(III) $Y$ is a geometric quotient of $X^{\prime}$ by $Y$. From the construction of $X^{\prime}$, we have an affine morphism $p^{\prime \prime}: X^{\prime} \rightarrow Y$ which is invariant with respect to the operation of $H$ on $X^{\prime}$. Therefore, there exists a geometric quotient $\left(Y^{\prime}, q\right): X^{\prime} \underset{q}{\rightarrow} Y^{\prime} \underset{q^{\prime}}{\rightarrow} Y, q^{\prime} \cdot q=p^{\prime}$.

Consider the following commutative diagram,

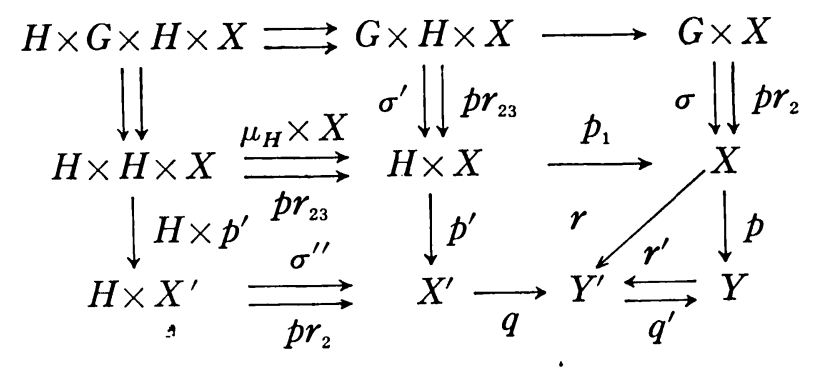

Since we have $q \cdot p^{\prime} \cdot\left(\mu_{H} \times X\right)=q \cdot \sigma^{\prime \prime} \cdot\left(H \times p^{\prime}\right)=q \cdot p r_{2} \cdot\left(H \times p^{\prime}\right)=$ $q \cdot p^{\prime} p r_{23}$, there exists a morphism $r: X \rightarrow Y^{\prime}$ such that $q \cdot p^{\prime}=r \cdot p_{1}$. We have $r \cdot \sigma=r \cdot p r_{2}$ by the analogous argument, hence the existence of a morphism $r^{\prime}: Y \rightarrow Y^{\prime}$ such that $r=r^{\prime} \cdot p$. Now, it is easy to show that $q^{\prime}, r^{\prime}$ define the isomorphisms of $Y^{\prime}$ and $Y$.

(IV) The proof of the second assertion can be done analogously. For the third assertion, we have a commutative diagram.

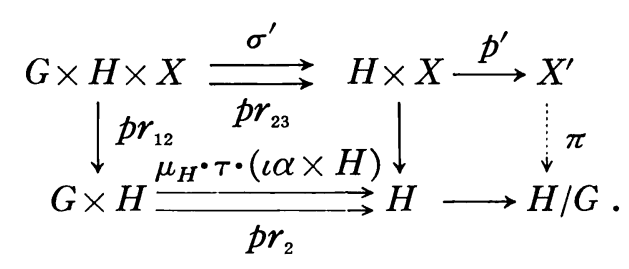


From this diagram, we know the existence of a $S$-morphism $\pi: X^{\prime} \rightarrow H / G$, where $H / G$ is a geometric quotient of $H$ by $G$, letting $G$ operate on $H$ from the right through $\iota \cdot \alpha$. Consider $\tilde{X}=\left(X^{\prime}, \pi\right) \underset{H / G}{\times}(S, e)$, where $e$ is the distinguished morphism induced by the unit morphism of $H . \quad \tilde{X}$ is a closed subscheme of $X^{\prime}$. From the construction of $X^{\prime}$, we know that $i: X \rightarrow X^{\prime}$ is divided by a canonical injection $j: \widetilde{X} \rightarrow X^{\prime}$. Using Lemma 1 of Part I, we know immediately that $X \cong \tilde{X}$.

q.e.d.

Corollary. Let $G$ be a commutative, finite, locally free group scheme over $S$ and let $Y$ be a scheme over $S$. Then $E_{S}(G, Y)$ is endowed with a structure of an abelian group. For a morphism $f: Z \rightarrow Y$, the mapping $f^{*}: E_{S}(G, Y) \rightarrow E_{S}(G, Z)$ is a homomorphism of abelian groups. For a homomorphism $\alpha: G \rightarrow H$ of commutative, finite, locally free group schemes, the morphism $\alpha_{*}: E_{S}(G, Y) \rightarrow$ $E_{S}(H, Y)$ is also a homomorphism of abelian groups.

Proof. Both the multiplication $\mu_{G}$ and the inverse morphism $\iota_{G}$ satisfy the condition $(S)$.

q.e.d.

\section{Part II. Main results}

1. From now on, $S$ is a spectrum of an algebraically closed field $k$ of arbitrary characteristic. Let $G$ be a finite group scheme over $k$ and let $X$ be an element of $E_{k}^{\prime}(G, Y)$, where $Y$ is a prescheme over $k$,

$$
G \times X \underset{p r_{2}}{\stackrel{\sigma}{\longrightarrow}} X \stackrel{p}{\longrightarrow} Y .
$$

Since $k$ is perfect, $(G \times \underset{k}{\times})_{\text {red }}=G_{\text {red }} \times X_{\text {red }}$, and since $\left(\sigma, p r_{2}\right): G \times \underset{k}{ } X$ $\rightarrow X \times \underset{k}{ } X$ is a closed immersion, $\left(\sigma_{\text {red }}^{k}, p r_{2}\right): G_{\text {red }} \times \underset{k}{*} X_{\text {red }} \rightarrow X_{\text {red }} \times{ }_{k}^{k} X_{\text {red }}$ is a closed immersion. Therefore $G_{\text {red }}$ operates faithfully on $X_{\text {red }}$. On the other hand, $p_{\text {red }}$ is affine and invariant with respect to the operation of $G_{\text {red }}$ on $X_{\text {red }}$. Hence the existence of a geometric quotient $\left(Y^{\prime}, p^{\prime}\right)$ of $X_{\text {red }}$ be $G_{\text {red }}$,

$$
G_{\mathrm{red}} \times X_{\mathrm{red}} \underset{p r_{2}}{\stackrel{\sigma_{\mathrm{red}}}{\longrightarrow}} X_{\mathrm{red}} \stackrel{p^{\prime}}{\longrightarrow} Y^{\prime} \stackrel{p^{\prime \prime}}{\longrightarrow} Y_{\mathrm{red}}, \quad p^{\prime \prime} \cdot p^{\prime}=p_{\mathrm{red}} .
$$


Then we have:

Lemma 1. In the above situation, the morphism $p^{\prime \prime}$ is a universal homeomorphism (cf. [4], IV, 2.4.2), and the homomorphism of structural sheaves attached to $p^{\prime \prime}: \Theta_{Y_{\mathrm{red}}} \rightarrow p_{*}^{\prime \prime}\left(\mathrm{O}_{Y^{\prime}}\right)$ (which we call the comorphism of $p^{\prime \prime}$ for the abbreviation of notations) is injective.

Proof. It is essentially included in the assertion (i) of Proposition 1, Part I. q.e.d.

Let $\alpha: F \rightarrow H, \beta: G \rightarrow H$ be homomorphisms of finite group schemes over $k$ and let $Y$ be a reduced, irreducible scheme of finite type over $k$ (i.e. an absolutely irreducible variety in the classical sense). Let $X$ be an element of $E_{k}^{\prime}(F \underset{H}{\times} G, Y) ;(F \underset{H}{\times} G) \underset{k}{\times}$

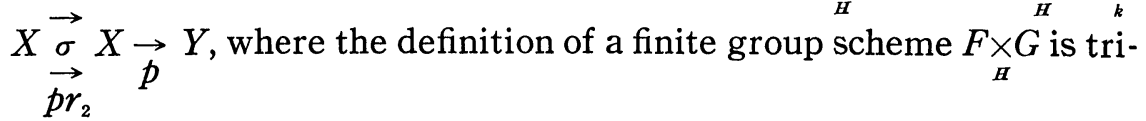
vial. Let $p_{1}, p_{2}, p_{3}$ be projections from $F \times \underset{H}{\times} G$ to $F, G$ and $H$. Consider $X_{1}=\left(p_{1}\right)_{*}(X), \quad X_{2}=\left(p_{2}\right)_{*}(X)$ and $X_{3}=\left(p_{3}\right)_{*}(X) ; F \underset{k}{\sigma_{H}} \underset{X_{1}}{\stackrel{\sigma_{F}}{\longrightarrow}} X_{1} \stackrel{p_{F}}{\rightarrow} Y$, $G \times X_{k} \underset{p r_{2}}{\stackrel{\sigma_{G}}{\rightrightarrows}} X_{2} \stackrel{p_{G}}{\rightarrow} Y, \quad H \times X_{3} \underset{p r_{3}}{\stackrel{\sigma_{H}}{\rightrightarrows}} X_{3} \stackrel{p_{H}}{\rightarrow} Y$. Then we have $\left(p_{3}\right)_{*}(X)=$ $\alpha_{*}\left(X_{1}\right)=\beta_{*}\left(X_{2}\right)$. Therefore, an element $\left(X_{1}, X_{2}\right)$ is determined in $E_{k}(F, Y) \underset{E_{k}(H, Y)}{\times} E_{k}(G, Y)$, associated to $X \in E_{k}(F \underset{H}{\times} G, Y)$. We denote this map by $\Phi$.

Lemma 2. The notations are as above. Then $\underset{X_{3}}{X_{X_{3}}} \underset{X_{2}}{X_{2}} \neq \phi$ and $X_{1} \underset{X_{3}}{\times} X_{2}$ is an element of $E_{k}(F \times \underset{H}{\times} G, Y)$.

Proof. It is easy to see that $X_{1} \times X_{3} \neq \phi$. We define an operation $\sigma^{\prime}$ of $\underset{H}{F} G$ on $X_{1} \times_{X_{3}} X_{2}$ as the composition of morphisms,

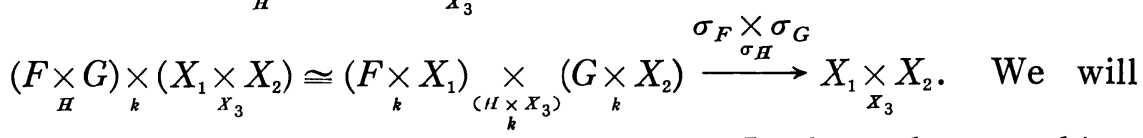
show that the operation $\sigma^{\prime}$ is faithfull. In fact, the morphism

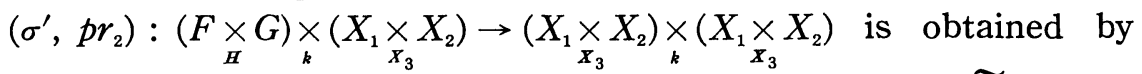

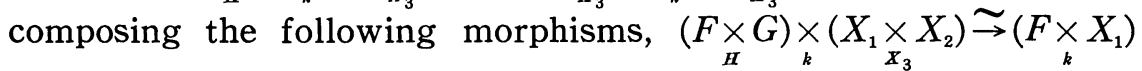

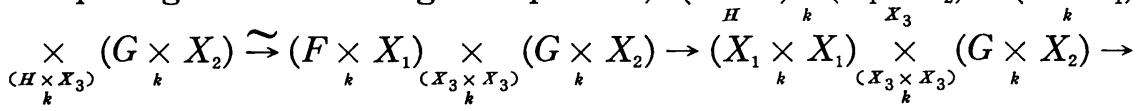


$\left(X_{1} \underset{k}{\times} X_{1}\right) \underset{\left(X_{3} \times X_{3}\right)}{\times}\left(X_{2} \times \underset{k}{\times} X_{2}\right) \underset{\sim}{\sim}\left(X_{1} \underset{X_{3}}{\times} X_{2}\right) \underset{k}{\times}\left(\underset{X_{3}}{X_{X_{3}}} \times X_{2}\right)$, where it is easy to point out the morphisms, (the second, $\left(F \times X_{k} X_{\left(\sigma_{H}, p r_{2}\right)} \underset{k}{\times}\left(G \times X_{2}\right)\right.$, etc.) and where all morphisms are closed immersions. Hence, $\left(\sigma^{\prime}, p r_{2}\right)$ is a closed immersion. Put $p^{\prime}=p_{F} \underset{p_{H}}{\times} p_{G}: X_{1} \times X_{X_{3}} \rightarrow Y$. Then $p^{\prime}$ is affine and satisfies $p^{\prime} \cdot \sigma^{\prime}=p^{\prime} \cdot p r_{2}$. Therefore we know the existence of a geometric quotient $\left(Y^{\prime}, q\right)$ of $\underset{X_{3}}{X_{1}} \times X_{2}$ by $F \underset{H}{\times} G$,

(*) $\quad(F \underset{H}{\times} G) \times \underset{k}{\times}\left(X_{X_{3}} \times X_{2}\right) \underset{p r_{2}}{\stackrel{\sigma^{\prime}}{\longrightarrow}}\left(X_{1} \times X_{X_{3}} X_{2}\right) \stackrel{q}{\longrightarrow} Y^{\prime} \stackrel{q^{\prime}}{\longrightarrow} Y, \quad q^{\prime} \cdot q=p^{\prime}$.

The base change of the diagram (*) by $p: X \rightarrow Y$ gives us a diagram,

$(* *) \quad(F \underset{H}{\times} G) \underset{k}{\times}(F \underset{H}{\times} G) \times \underset{k}{X} \underset{p r_{2}}{\stackrel{\mu^{\prime} \times X}{\longrightarrow}}(F \times \underset{H}{\times} G) \times \underset{k}{\longrightarrow} X \rightarrow\left(Y^{\prime} \underset{Y}{\times} X\right) \stackrel{q_{X}^{\prime}}{\longrightarrow} X$,

where we use the relation, $\left(X_{1} \underset{X_{3}}{\times} X_{2}\right) \underset{Y}{\times} \underset{\longrightarrow}{\sim} \underset{\rightarrow}{\sim}\left(X_{1} \underset{Y}{\times} \underset{\left(X_{3} \times X\right)}{\times}\left(X_{Y} \times \underset{Y}{\times} X\right)\right.$ $\widetilde{\sim}\left\{\left(X_{1} \underset{Y}{\times} X_{1}\right) \underset{X_{1}}{\times} X\right\} \underset{\left(\left(X_{3} \times X_{Y} X_{3}\right) \times X X\right.}{\times}\left\{\left(X_{2} \times \underset{Y}{\times} X_{2}\right) \underset{X_{2}}{\times} X\right\} \underset{\rightarrow}{\sim}(F \times \underset{k}{\times} X) \underset{(H \times X)}{\times}(G \underset{k}{\times} X) \stackrel{\sim}{\rightarrow}$ $(F \underset{H}{\times} G) \underset{k}{\times} X$ and where $\mu^{\prime}$ is the multiplication of $F \underset{H}{\times} G$. From (**), we know that $q_{X}^{\prime}: Y_{Y}^{\prime} \underset{Y}{\sim} \underset{\rightarrow}{\sim} \underset{Y}{\sim} X$. Since $p: X \rightarrow Y$ is a faithfully flat, quasi-compact morphism, we know that $q^{\prime}: Y^{\prime} \sim \mathcal{\rightarrow}$. q.e.d.

Lemma 3. The notations are as in Lemma 2. We put the next condition.

(T). Let $\xi($ resp. $\eta)$ be any element of $E_{k}(F, Y)\left(\right.$ resp. $\left.E_{k}(G, Y)\right)$ such that $\alpha_{*}(\xi)=\beta_{*}(\eta)$. Then we can find $X_{1}, X_{2}, X_{3}$ respectively in the classes $\xi, \eta$ and $\alpha_{*}(\xi)=B_{*}(\eta)$, such that the canonical images of $X_{1}$ and $X_{2}$ in $X_{3}$ have non-empty intersection and that the images of $X_{1} \times X_{X_{3}}$ in $Y$ contains a generic point of $Y$ over $k$.

Then we can define a mapping $\Psi: E_{k}(F, Y) \underset{B_{k}(1, Y, Y)}{\times} E_{k}(G, Y) \rightarrow$ $E_{k}(F \times G, Y)$ by $(\xi, \eta) \rightarrow$ the class of $\left(X_{1} \times X_{x_{3}}\right)$ and $\Psi$ is an isomorphism, i.e. $\Phi \cdot \Psi=1, \Psi \cdot \Phi=1$.

Proof. The proof consists of several steps.

(I) If either $\alpha$ or $\beta$ is epimorphic: Suppose $\alpha$ is epimorphic. 
Take arbitrarily $X_{1}, X_{2}$ in $\xi, \eta$. (The same notations as in the assumption $(T)$ ). Then, as $X_{3}$, we can choose a geometric quotient $\left(X_{1} / K, i_{1}\right)$, where $K$ is a kernel of $\alpha$. Thus $i_{1}: X_{1} \rightarrow X_{3}$ is finite, locally free, hence faithfully flat, quasi-compact and immediately $X_{1} \times X_{3} \neq \phi$. The proof of the facts that the operation $\sigma^{\prime}$ of $\underset{H}{F} G$ on $X_{1} \times X_{3}$ is well defined, faithfull and that there exists a geometric quotient $\left(Y^{\prime}, q\right)$ of $\underset{X_{3}}{X_{1}} \times X_{2}$ by $\underset{H}{F} G$ is analogous to the proof in Lemma 2. Thus we have a diagram,

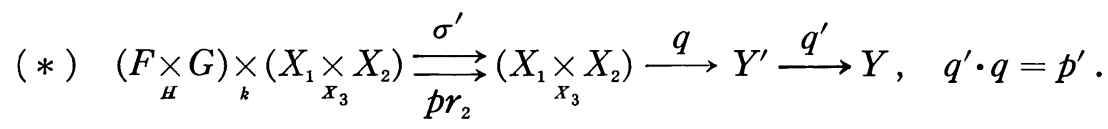

Note that $p^{\prime}=p_{F} \underset{p_{H}}{\times} p_{G}: X_{X_{3}} \times X_{2} \rightarrow Y$ is faithfully flat, quasi-compact because $i_{1}: X_{1} \rightarrow X_{3}$ and $p_{G}: X_{2} \rightarrow Y$ are also f.p.q.c. morphisms. To show $Y^{\prime} \underset{q^{\prime}}{\stackrel{\longrightarrow}{\longrightarrow}} Y$, we have only to change the base $Y$ of $(*)$ to $X_{1} \times X_{X_{3}}$ by $p^{\prime}: X_{1} \times X_{X_{3}} \rightarrow Y$. It is immediate to see that the class of $X_{1} \times X_{3}$ is independent of the choice of $X_{1}, X_{2}$ in $\xi, \eta$.

(II) The homomorphisms $\alpha$ and $\beta$ are decomposed as follows, $\alpha: F \stackrel{\alpha^{\prime}}{\rightarrow} F^{\prime} \stackrel{\alpha^{\prime \prime}}{\rightarrow} H, \beta: G \stackrel{\beta^{\prime}}{\rightarrow} G^{\prime} \stackrel{\beta^{\prime \prime}}{\rightarrow} H$, where $\alpha^{\prime}, \beta^{\prime}$ are epimorphic and $\alpha^{\prime \prime}$, $\beta^{\prime \prime}$ are monomorphic (hence, closed immersions). Therefore, by virtue of (I), it is easy to see that we have only to prove Lemma 3 in the case that $\alpha$ and $\beta$ are closed immersions.

(III) If both $\alpha$ and $\beta$ are closed immersions: Take $X_{1}, X_{2}, X_{3}$ as in the assumption (T). Then canonical morphisms $i_{1}: X_{1} \rightarrow X_{3}$ and $i_{2}: X_{2} \rightarrow X_{3}$ are closed immersions. Therefore we can consider that $X_{1}$ and $X_{2}$ are closed subschemes of $X_{3}$. Consider $\left(X_{1}\right)_{\text {red }}$, $\left(X_{2}\right)_{\text {red }}$ and $\left(X_{3}\right)_{\text {red }}$. $\left(X_{1}\right)_{\text {red }}$ and $\left(X_{2}\right)_{\text {red }}$ are closed subsets of the algebraic set $\left(X_{3}\right)_{\text {red }}$ and they have the same dimension as $\left(X_{3}\right)_{\mathrm{red}}$. Therefore $\left(X_{1}\right)_{\text {red }}$ and $\left(X_{-}\right)_{\text {red }}$ are the unions of some irreducible components of $\left(X_{3}\right)_{\mathrm{red}}$. Since $p^{\prime}: X_{1} \times_{X_{3}} X_{2} \rightarrow Y$ is propre and since the image of $p^{\prime}$ contains a generic point $y$ of $Y$ over $k$, the image of $p^{\prime}$ coincides with $Y$. From the assumption, $\left(X_{1} \cap X_{2}\right)_{\text {red }}$ contains a generic point of $\left(X_{3}\right)_{\text {red }}$ over $k$, hence an irreducible component. 
Therefore $\left(X_{1} \cap X_{2}\right)_{\text {red }}$ is the union of some irreducible components of $\left(X_{3}\right)_{\mathrm{red}}$. In fact, it is also the union of some connected components of $\left(X_{3}\right)_{\text {red }}$. Then the comorphism of $p^{\prime}: \mathcal{O}_{Y} \rightarrow p_{*}^{\prime}\left(\mathcal{O}_{X_{X_{3}} X_{2}}\right)$ is injective. On the other hand, $F \times{ }_{H} G$ operates faithfully on $X_{1} \times X_{2}$, and $p^{\prime}$ is affine and invariant with respect to the operation of $F \times G$. Thus we know the existence of a geometric quotient $\left(Y^{\prime}, q\right)$,

$$
\text { (*) } \quad(F \underset{H}{\times} G) \underset{k}{\underset{k}{*}\left(X_{1} \times \underset{X_{3}}{\times} X_{2}\right) \longrightarrow} \longrightarrow \underset{X_{3}}{\longrightarrow}\left(X_{1} \times X_{2}\right) \stackrel{q}{\longrightarrow} Y^{\prime} \stackrel{q^{\prime}}{\longrightarrow} Y, \quad q^{\prime} \cdot q=p^{\prime} .
$$

From the construction of $Y^{\prime}$, it is evident that the comorphism of $q^{\prime}: O_{Y} \rightarrow q_{*}^{\prime}\left(O_{Y^{\prime}}\right)$ is injective.

(IV) For any $k$-morphism $T \rightarrow Y$, we know that $\left(X_{3} \times T\right) \cong$ $\alpha_{*}\left(X_{1} \times T\right) \cong \beta_{*}\left(X_{2} \underset{Y}{\times} T\right)$ and that $Y^{\prime} \times T$ is a geometric quotient

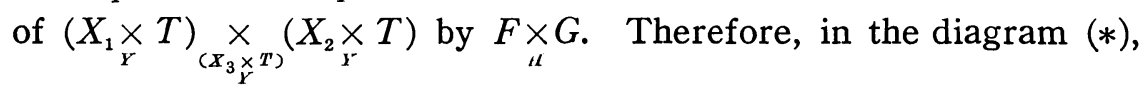
we can suppose, first $Y$ is affine, then $Y$ is a spectrum of a local ring and then $Y$ is a spectrum of a field $K$. In the last case, it is immediate to see that $X_{1} \times X_{3}$ is faithfully flat, quasi-compact over $Y$. Then the base change of $(*)$ by $p^{\prime}: X_{1_{3}} \times_{X_{3}} \rightarrow Y$ shows

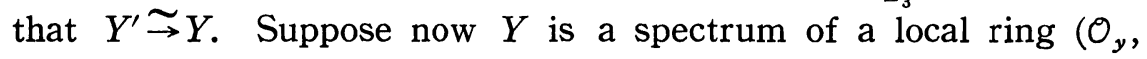
$\left.\mathfrak{M}_{y}\right)$ of some point $y$ of the original $Y$. Put $\left(\mathcal{O}_{y}, \mathfrak{M}_{y}\right)=(B, \mathfrak{M})$. Then $Y^{\prime}$ is a spectrum of $B$-algebra $B^{\prime}$ which is finite $B$-module and $B$ is a subalgebra of $B^{\prime}$ by virtue of the fact that $O_{Y}$ contained in $q_{*}^{\prime}\left(\mathcal{O}_{Y^{\prime}}\right)$. From the fact that $Y^{\prime} \otimes_{B} B / \mathfrak{M} \cong Y \otimes_{B} B / \mathfrak{M} \cong$ Spece $(B / \mathfrak{M})$, we know that $B^{\prime} / \mathfrak{M} B^{\prime} \sim B / \mathfrak{M}$, hence $B^{\prime}=B+\mathfrak{M} B^{\prime}$. Therefore $B^{\prime}=B$ by Nakayama's lemma, hence $Y^{\prime} \underset{q}{\stackrel{\sim}{q}} Y$. Thus $X_{1} \times X_{X_{3}} X_{2} \in E_{k}^{\prime}(F \times \underset{H}{\times} G, Y)$. It is immediate to see that the class of $X_{1} \times X_{3}$ depends only on the classes $\xi$ and $\eta$.

(V) Put $X_{1} \underset{\boldsymbol{x}_{3}}{\times} X_{2}=X$. Consider a morphism $\sigma_{F} \cdot r: F \underset{k}{ }{ }_{k} \rightarrow X_{1}$, where $r$ is the projection $F \underset{k}{F}\left(X_{1} \times X_{X_{3}}\right)$ to $F \times X_{k}$. Then $\sigma_{F} \cdot r$ is invariant with respect to the operation of $\underset{H}{F}$ 作 $F \times X$ which 
is defined in Proposition 3 of Part I. Therefore we have a morphism $\lambda:\left(p r_{1}\right)_{*}(X) \rightarrow X_{1}$ which is compatible with the operations of $F$ and which makes the following diagram commutative,

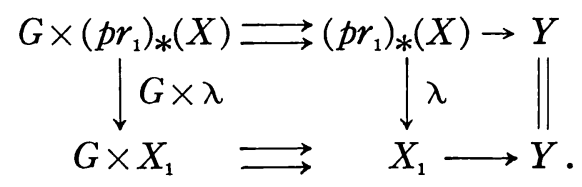

Hence $\left(p r_{1}\right)_{*}(X) \stackrel{\lambda}{\stackrel{\lambda}{\rightarrow}} X_{1}$. The same argument for $p r_{2}: F \underset{H}{\times} G \rightarrow G$ shows us $\left(p r_{2}\right)_{*}(X) \stackrel{\sim}{\rightarrow} X_{2}$. q.e.d.

Lemma 4. The notations are as in Lemma 3. The condition of Lemma 3 is satisfied in the following cases.

(i) Either $\alpha$ or $\beta$ is epimorphic.

(ii) Either $F$ or $G$ is the unit group scheme $\operatorname{Spec}(k)$.

(iii) $F, G, H$ are commutative.

(iv) $F, G, H$ are infinitesimal (i.e. whose affine rings are local.)

Proof. (i) is trivial.

(ii) Suppose $G=\operatorname{Spec}(k)$. Then any element of the class $\eta$ is isomorphic to $Y$. Take $X_{1}, X_{2}, X_{3}$ arbitrarily in $\xi, \eta$ and $\alpha_{*}(\xi)=$ $\beta_{*}(\eta)$. Then $i_{2}: X_{2} \rightarrow X_{3}$ is a section $s: Y \rightarrow X_{3}$ to $p_{H}$. Take generic points $x_{1}, x_{2}$ of $\left(X_{1}\right)_{\text {red }}$ and $X_{2}$ over $k$ such that $x_{1}, x_{2}$ belong to the same orbit by $H_{\text {red }}$. (It is possible by virtue of Lemma 1.) Then there exists an element $h$ of $H_{\text {red }}$ such that $x_{1}=h x_{2}$, where $h$ is $k$-rational. Now take $X_{2}^{\prime}=h X_{2}$ in $\eta$, (it corresponds to a section $h s$ ). These $X_{1}, X_{2}, X_{3}$ satisfy the condition $(T)$.

(iii) The analogous argument to (ii) is applicable in this case.

(iv) Take $X_{1}, X_{2}, X_{3}$ arbitrarily in $\xi, \eta$ and $\alpha_{*}(\xi)$. Then $X_{1}$, $X_{2}, X_{3}$ satisfy the condition $(T)$.

q.e.d.

We put the following notations:

$C_{f}^{c}(k)=$ the category of commutative finite group schemes over $k$. $C_{f}^{\inf }(k)=$ the category of finite group schemes whose affine rings are local.

Then we have :

Theorem. Suppose $Y$ is an irreducible variety over $k$. Then we have: 
(i) If $\alpha: F \rightarrow G$ is a monomorphism of finite group schemes, then the map of sets $\alpha_{*}: E_{k}(F, Y) \rightarrow E_{k}(G, Y)$ is injective,

(ii) Let (1) $\rightarrow K \underset{\alpha}{\rightarrow} F_{\beta} G \rightarrow(1)$ be an exact sequence of finite group schemes, i.e. $K$ is a normal subgroup of $F$ and $G \cong F / K$. Then in the sequence,

$$
E_{k}(K, Y) \stackrel{\alpha_{*}}{\longrightarrow} E_{k}(F, Y) \stackrel{\beta_{*}}{\longrightarrow} E_{k}(G, Y)
$$

if the image of an element $\eta$ of $E_{k}(F, Y)$ by $\beta_{*}$ is trivial, then there exists an element $\xi$ of $E_{k}(K, Y)$ such that $\alpha_{*}(\xi)=\eta$.

(iii) A covariant functor $G \longrightarrow E_{k}(G, Y)$ from the category $C_{f}^{c}(k)$ into the category of abelian groups is strictly pro-representable.

(iv) A covariant functor $G \longrightarrow E_{k}(G, Y)$ from the category $C_{f}^{\mathrm{inf}}(k)$ into the category of sets is strictly pro-representable.

Proof. Use Lemma 3, Lemma 4 and Grothendieck's theorem for the pro-representability, [3], $\mathrm{n}^{\circ} 195-06$.

Remark. The results (i), (ii) of Theorem hold for $Y$ which is a connected, reduced scheme of finite type over $k$.

2. As in 1 of Part II, $S$ is a spectrum of an algebraically closed field $k$ of arbitrary characteristic and $Y$ is a reduced, irreducible scheme of finite type over $k$. Let $y$ be a generic point of $Y$ over $k$ and let $X$ be an element of $E_{k}^{\prime}(G, Y)$, where $G$ is a finite group scheme over $k$. Consider a fibre $X_{y}=\underset{Y}{\times} \boldsymbol{k}(y)$. It is a principal homogeneous space under $G$ defined over $\boldsymbol{k}(y)$. Take a geometric point $x$ of $X_{y}$ and consider a pair $(X, x)$ (i.e. $X$ with the ponctuation). When we consider a morphism of such ponctuated preschemes, we add the condition that the morphism preserves the ponctuations. Then Lemma 1 of Part I holds for the case that $X$ and $X^{\prime}$ are ponctuated. We denote by $E_{k}(G ; Y, y)$ the quotient set of $E_{k}^{\prime}(G, Y)$ by the analogous equivalence relation to the one which defines $E_{k}(G, Y)$. Then $G_{\text {red }}$ operates on $E_{k}(G ; Y, y)$ by translating the ponctuations $x \cdots g x$, and $E_{k}(G ; Y, y) / G_{\text {red }}$ is canonically isomorphic to $E_{k}(G, Y)$. Note that when we construct $\alpha_{*}(X)$ for $\alpha: G \rightarrow H$, we can endow $\alpha_{*}(X)$ with a canonical ponctuation i.e. the class of $(1, x)$ modulo $G$. Then all results of Part 
I and Lemma 1, Lemma 2 of Part II hold for the ponctuated preschemes and for the morphisms of ponctuated preschemes. In Lemma 3 , the condition is naturally satisfied. Therefore for homomorphisms of finite group schemes $\alpha: F \rightarrow H$ and $\beta: G \rightarrow H$, we have an isomorphism of sets,

$$
E_{k}(F \times \underset{H}{\times} G ; Y, y) \stackrel{\longrightarrow}{\longrightarrow} E_{k}(F ; Y, y)_{B_{k}(H ; Y, y, y)}^{\times} E_{k}(G ; Y, y) .
$$

Therefore, by virtue of Grothendieck's Theorem for the prorepresentability, a covariant functor $G \longrightarrow E_{k}(G ; Y, y)$ from the category of finite group schemes $C_{f}(k)$ to the category of sets is strictly pro-representable.

Remark. If $G$ belongs to $C_{f}^{c}(k), E_{k}(G ; Y, y) \rightarrow E_{k}(G, Y)$ because $G_{\text {red }}$ operates trivially on $E_{k}(G ; Y, y)$. Also, if $G$ belongs to $C_{f}^{\inf }(k)$, $E_{k}(G ; Y, y)=E_{k}(G, Y)$ because $G_{\text {red }}=\operatorname{Spec}(k)$.

\section{Part III. Appendix.}

1. As in Part II, $S$ will be a spectrum of an algebraically closed field $k$ of arbitrary characteristic. Let $A$ be an abelian variety defined over $k$ and let $G$ be a finite, commutative group scheme over $k$ which operates faithfully on a connected, reduced prescheme $X$ over $k$ and gives a geometric quotient $A$,

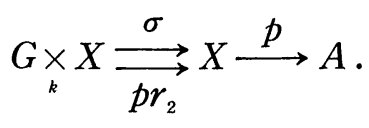

In this Part, we will prove that $X$ is necessarily an abelian variety and that $p$ is an isogeny. This result is a slight generalization of the result of Lang-Serre on the non-ramified coverings of an abelian variety, [9].

2. Since $G$ is commutative, $G$ is a direct product of a reduced subgroup scheme $G_{\text {red }}$ and an infinitesimal subgroup scheme $G_{\text {inf }}$, $G=G_{\text {red }} \cdot G_{\text {inf }}$. By the process of Proposition 3 of Part I, we have an element $X^{\prime} \in E_{k}^{\prime}\left(G_{\text {red }}, A\right)$ and a diagram, 


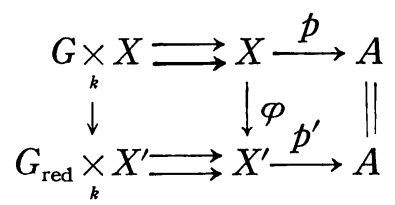

where $X^{\prime} \cong G_{\text {red }} \times X / G \cong X / G_{\text {inf }}$ and where $X^{\prime}$ is connected, reduced. Then, noting that $G_{\text {red }} \cong G(k)_{k}$ and combining the results of Grothendieck, [2], Exp. V, Prop. 2. 6 and Cor. 2. 4, we know $p^{\prime}: X^{\prime} \rightarrow A$ is an étale covering of $A$. And by the results, [2], Exp. I, Prop. 9.2, Theorem 9.5. (i) and [4], II, 6.1.10, $p^{\prime}: X^{\prime} \rightarrow A$ is a nonramified covering of $A$ in the sense of Lang-Serre, [9]. Therefore, we know from Theorem of Lang-Serre, loc. cit. that $X$ is an abelian variety and that $p$ is a separable isogeny. Now, since $G_{\text {inf }} \times X \rightrightarrows \rightarrow \stackrel{\mathscr{P}}{\rightarrow} X^{\prime}$ is exact, we can suppose from the first that $k$ is of positive characteristic $p$ and that $G$ is an infinitesimal commutative group scheme over $k$. On the other hand, by the general theory of commutative group schemes, we know the existence of a closed subgroup scheme $G^{\prime}$ of $G$ such that the quotient $G / G^{\prime}$ is a simple object in the category of commutative group schemes, i.e. $G / G^{\prime}=\alpha_{p}$ or $\mu_{p}$, cf. [10]. $\alpha_{p}$ (resp. $\mu_{p}$ ) is obtained as the kernel of the Frobenius endomorphism $\boldsymbol{p}$ of the additive (resp. multiplicative) group $G_{a}$ (resp. $G_{m}$ ).*) It is easy to see that we have only to prove our result in the case that $G=\alpha_{p}$ or $\mu_{p}$. As above, $\alpha_{p}$ (resp. $\mu_{p}$ ) is a finite subgroup of $G_{a}$ (resp. $G_{m}$ ). The process of Proposition 3 of Part I is here applicable to obtain a principal fibre space $X^{\prime \prime}$ of the base $A$ and of the group $G_{a}$ (resp. $G_{m}$ ) from $X$, here a principal fibre space is the one in the sense of f.p.q.c. topology,
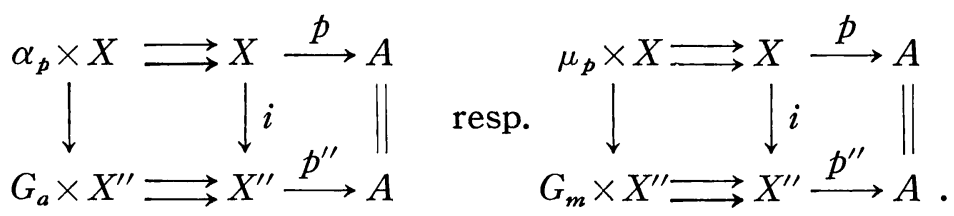

It is easy to see that a canonical injection $i: X \rightarrow X^{\prime \prime}$ is a closed immersion, that $X^{\prime \prime}$ is a connected, reduced, moreover that $X^{\prime \prime}$ is an

(*) The idea to embed $\alpha_{p}$ (resp. $\mu_{p}$ ) into $G_{a}$ (resp. $\mathrm{G}_{m}$ ) and to use the results of Rosenlicht and Serre was suggested by T, Oda. 
irreducible variety. Let $\xi$ be a generic point of $A$ over $k$, and consider a fibre $X_{\xi}^{\prime \prime}=X^{\prime \prime} \times \underset{A}{\times}(\xi)$. Then $X_{\xi}^{\prime \prime}$ is a principal homogeneous space with respect to $G_{a}$ (or $G_{m}$ ) defined over the field $\boldsymbol{k}(\xi)$. Therefore Lemma for Theorem 10 of [7] shows us that $X_{\xi}^{\prime \prime}$ has a $k(\xi)$-rational point $\eta$. By associating the point $\eta$ to the point $\xi$, we have a rational section $s$ to $p^{\prime \prime}, s: A \rightarrow X^{\prime \prime}$ which is regular at $\xi$. Since $A$ is a commutative group variety, $X^{\prime \prime}$ is a locally trivial principal fibre space of the base $A$ and of the group $G_{a}$ (or $G_{m}$ ), i.e. the one in the sense of Zariski topology. Thus $X^{\prime \prime}$ belongs to one class of $H^{1}\left(A, O_{A}\right)$ (or $H^{1}\left(A, O_{A}^{*}\right)$ ). From the construction of $X^{\prime \prime}$, it is easy to see that the class of $X^{\prime \prime}$ in $H^{1}\left(A, \mathcal{O}_{A}\right)$ (or $\left.H^{1}\left(A, \mathcal{O}_{A}^{*}\right)\right)$ is annihilated by the multiplication by $p$, the characteristic of the field $k$. From the Serre's book, [8], we know that $H^{1}\left(A, O_{A}\right) \cong \operatorname{Ext}\left(A, G_{a}\right)$ and that the torsion elements of $\left.H^{1}\left(A, O_{A}^{*}\right)\right\} \subset \operatorname{Ext}\left(A, G_{m}\right)$. Therefore in both cases, we can consider that $X^{\prime \prime}$ is a commutative group variety. Since $i: X \rightarrow X^{\prime \prime}$ is a closed immersion and since $X$ is propre over $k, X$ can be considered as a complete subvariety of $X^{\prime \prime}$ of codimension 1 which contains a unit element $e$ of $X^{\prime \prime}$. It is not difficult to see that the algebraic group $G(X)$ which is generated by $X$ in $X^{\prime \prime}$ is closed, connected and complete. Hence $G(X)$ is an abelian sub-variety of $X^{\prime \prime}$. Since there is a connected linear group $G_{a}$ (or $G_{m}$ ) of dimension 1 in $X^{\prime \prime}, G(X)$ is a closed subgroup of codimension 1 and contains $X$. Hence $G(X)$ coincides with $X$. Therefore $X$ is an abelian variety. Thus we have:

Theorem. Let $G$ be a commutative finite group scheme ever an algebraically closed field $k$ of arbitrary characteristic, and let $X$ be a connected, reduced k-prescheme over which $G$ operates faithfully and gives a geometric quotient $A$,

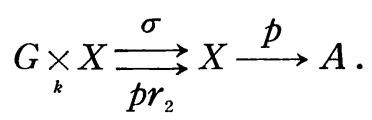

Then $X$ is an abelian variety and $p$ is an isogeny. 


\section{BIBLIOGRAPHY}

[1] M. Demazure et A. Grothendieck, S.G.A.D. 1963/64, Exposés II, IV, V, VI, VII, VIII, X. Mimeographed notes of I.H.E.S.

[2] A. Grothendieck, S.G.A., 1961 Mimeographed note of I.H.E.S.

[3] A. Grothendieck, Fondements de la géométrie algébrique (extraits du Séminaire Bourbaki 1957-1962). Paris, 1962.

[4] A. Grothendieck et J. Dieudonné, Éléments de Géométrie Algébrique, Chap. I, II, IV ${ }^{2}$, Publ. Math. de I.H.E.S.

[5] D. Mumford, Geometric Invariant Theory, Ergebnisse Math., Bd. 24, Springer Verlag, 1965.

[6] M. Miyanishi, La pro-représentabilité d'un foncteur sur la catégorie des groupes formels artiniens, C.R. Acad. Sc. Paris, t. 262, 1966.

[7] M. Rosenlicht, Some basic theorems on algebraic groups, Amer. J. Maths., 78, 1956, 401-443.

[8] J.-P.-Serre, Groupes algébriques et corps de classes, Hermann, Paris, 1959.

[9] S. Lang et J.-P.-Serre, Sur les revêtements non-ramifiés des variétés algébriques, Amer. J. Maths., 79, 1957, 319-330.

[10] F. Oort, Commutative group schemes, Lecture Notes in Maths., $\mathrm{N}^{\circ}{ }_{1}$, Springer Verlag, 1966. 